\section{SMART ${ }^{3}$ Handpiece Decontamination Protocol launched}

As the UK's market leader in handpiece and decontamination technology, NSK is committed to improving the standards of handpiece decontamination, especially in light of the heightened risks posed by transmission of harmful pathogens, particularly by asymptomatic patients.

Until now, the risk from dental handpieces has tended to focus on the issues surrounding aerosol production. However, little has been said about the importance of the need to correctly decontaminate handpieces which is vital in helping to prevent the risk of cross-infection between patients.

NSK's SMART ${ }^{3}$ handpiece decontamination protocol consists of three key steps - Cleaning, Lubrication \& Sterilisation - and, when using a proven, automated system, provides the following benefits:

$\rightarrow$ Safer - compared with inconsistent manual processes and the use of incorrect autoclave/sterilisation cycles

$\rightarrow$ Measured - employing proven systems that dose the correct volumes of cleaning solution and lubricant, handpieces can be sterilised using the correct autoclave cycles $-\mathrm{S}$ or B type vacuum

$\rightarrow$ Affordable - ensures the overall process will deliver a return on investment based

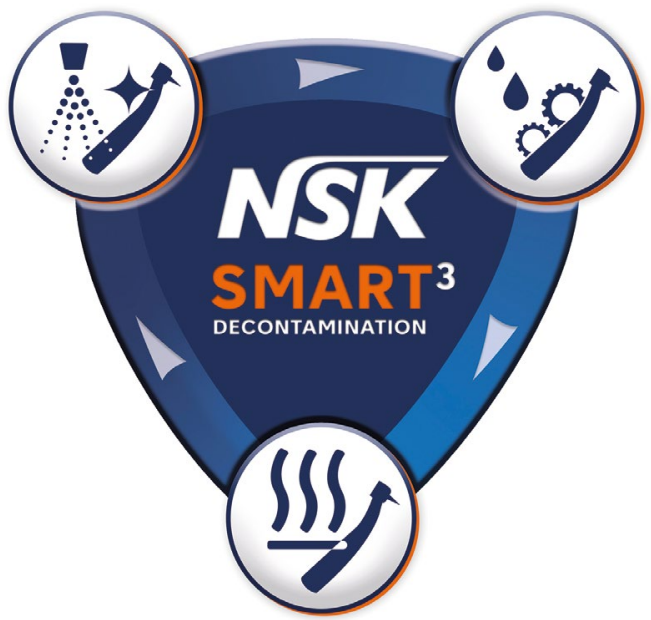

on the cost savings of the consumables used, over-reliance on expensive inventory and, ongoing handpiece maintenance costs

$\rightarrow$ Reproducible - provides consistent results, time after time, by removing the ambiguities associated with a manual process

$\rightarrow$ Time saving - significantly decreases the time required to manually decontaminate handpieces, helping improve throughput and reducing costly delays in the process.

To discover more about NSK's wide range of decontamination equipment and the NSK SMART ${ }^{3}$ protocol visit mynsk. co.uk/smart3/

\title{
See the future
}

Help future proof your practice by outsourcing payroll to premium provider Wagemate.

It doesn't matter if your practice is big or small, you will save time, money and stress with Wagemate.

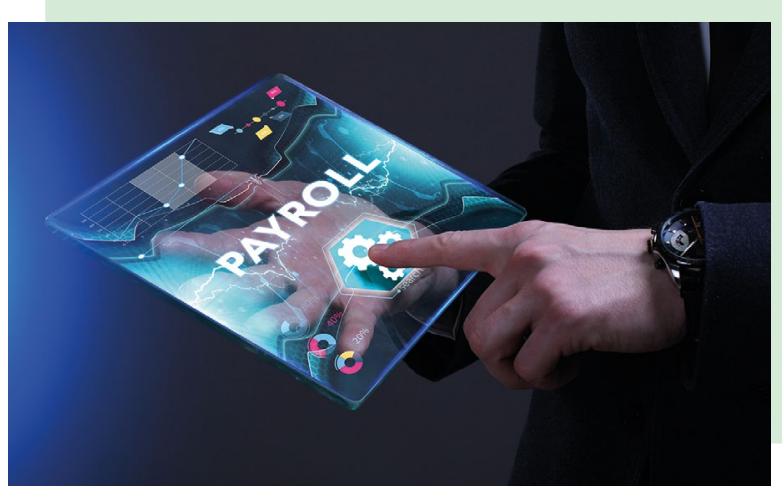

Everyone will be paid correctly, and on time. You can focus on developing your business, with peace of mind that if you need to upscale your payroll, or the rules change, there is no problem that the experts at Wagemate cannot solve, and fast! Imagine payroll taken off your hands, with you staying fully compliant - with Wagemate you'll be one step ahead.

To better manage your payroll, contact payroll specialists Wagemate on 03330102102 or email info@ wagemate.com.

\section{Love your gloves!}

Gloves form such an important part of your PPE in practice. But are the gloves you are currently using best suited to your team?

Gloves need to fit correctly in order to provide the best level of protection and not cause any allergies in those with sensitive skin or who react to latex. Plus, they can be a great extension of your practice identity if you choose colours and styles that fit your brand.

Initial Medical has a comprehensive selection of gloves available that ensures you can always find solutions suited to the needs of your team. From latex-free options for those who suffer allergies to gloves available in a range of colours and sizes, you can make sure your gloves provide exceptional protection and enhance the identify of your practice

For further information visit www.initial. co.uk/medical or call 08708504045 .

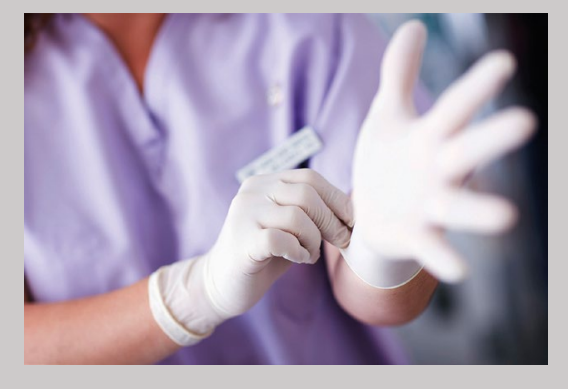

\section{Bolster your dental practice}

Ten Dental+Facial's Implant Restoration Course (IRC) offers dentists the chance to expand their skill-set and bring dental implant treatment to their practices.

Over the course of the year, you'll learn the process of restoring implants under the expert guidance of Drs Nikhil Sisodia and Martin Wanendeya, taught through both theory and hands-on. The modules will teach you how to diagnose, plan, and restore implants in your own practice.

Even with no prior implant experience, you will be offering your patients implant restorations in both simple and complex cases in no time.

For more information visit www. tendental.com, email referrals@tendental. com or call on 02086751798 . 